\title{
天然黄酮香叶木素类及其衍生物的合成与生物活性研究
}

\author{
蔡双莲 ${ }^{a}$ 吴 峥 ${ }^{a}$ 吴 进 ${ }^{a}$ \\ 汪秋安 $*$, 单 杨 ${ }^{b}$ \\ ( ${ }^{a}$ 湖南大学化学化工学院 长沙 410082$)$ \\ ( 湖南省柑桔工程技术研究中心 长沙 410125)
}

\begin{abstract}
摘要 以橙皮苷为原料, 经脱氢、选择性甲基化、糖苷水解、相转移催化下的糖苷化反应、异戍烯基化和法呢烯基化 等反应步骤, 分别合成了 $3^{\prime}-O$-甲基香叶木素(1), 香叶木素-7-O- $\beta-D$ - 葡萄糖苷(2), 香叶木素-7- $O-\beta-D$-半乳糖苷 $(3), 3^{\prime}-O-$ 甲基香叶木素-7-O- $\beta-D$ - 葡萄糖苷(4) 4 种天然产物及 $3^{\prime}-O$-甲基香叶木素-7-O- $\beta-D$-半乳糖苷(5), 香叶木素-7-O- $\beta-D-$ 乙酰 葡萄糖苷(6)、香叶木素-7-O- $\beta$ - $D$-乙酰半乳糖苷(7), $3^{\prime}-O$-甲基香叶木素-7- $O-\beta$ - $D$-乙酰葡萄糖苗(8), $3^{\prime}-O$-甲基香叶木素7- $O-\beta$ - $D$-乙酰半乳糖苷 (9), 7- $O$-异戊基香叶木素(10), 7- $O$-异戊烯基-3'- $O$-甲基香叶木素(11)和 7- $O$-法呢烯基-3'- $O$-甲基香 叶木素(12) 8 种新的香叶木素衍生物. 所合成化合物的结构已由核磁共振谱、红外光谱和质谱所证实, 并用比色法 MTT [3-(4,5-二甲基噻唑-2)-2,5-二苯基四氮唑溴盐]蛋白染色法对所合成的目标化合物进行了体外抗肿瘤细胞生物活性测试, 发现化合物 6, 10 和 11 对肝癌细胞(SMMC-7721)、乳腺癌细胞(MCF-7)和结肠癌细胞(SW480)有一定的抑制活性.
\end{abstract}

关键词 黄酮类; 橙皮苷; 香叶木素; 合成; 生物活性

\section{Synthesis and Biological Activities of Natural Flavonoid Diosmetin and Its Derivatives}

\author{
Cai, Shuanglian $^{a} \quad{\mathrm{Wu}, \text { Zheng }^{a} \quad \mathrm{Wu}^{\text {. Jing }}{ }^{a} \quad \text { Wang, Qiuan }^{*, a} \quad \text { Shan, Yang }}^{b}$ \\ $\left({ }^{a}\right.$ College of Chemistry and Chemical Engineering, Hunan University, Changsha 410082) \\ $\left({ }^{b}\right.$ Centre for Citrus Engineering Technology Research in Hunan, Changsha 410125)
}

\begin{abstract}
Four natural flavonoids 3'-O-methyldiosmetin (1), diosmetin-7-O- $\beta$-D-glucoside (2), diosmetin-7- $O-\beta-D$-galactoside (3), 3'-O-methyldiosmetin-7-O- $\beta$-D-glucoside (4), and eight new diosmetin derivatives 3'-O-methyldiosmetin-7$O-\beta$ - $D$-galactoside (5), diosmetin-7-O- $\beta$ - $D$-acetylglucoside (6), diosmetin-7-O- $\beta$ - $D$-acetylgalactoside (7), $3^{\prime}-O$-methyl- diosmetin-7-O- $\beta$ - $D$-acetylglucoside (8), 3'-O-methyldiosmetin-7-O- $\beta$ - $D$-galactoside (9), 7-O-isopentyldiosmetin (10), 7-O-prenyldiosmetin (11) and 7-O-farnesyl-3'-O-methyldiosmetin (12) were synthesized from hesperidin by reaction steps including dehydrogenation, selective methylation, glycoside hydrolysis, glycosylation under phase transfer catalytic condition, $O$-prenylation and $O$-farnesylation, respectively. Their structures were confirmed by NMR, IR and MS techniques. The synthesized compounds were evaluated for biological activity against four human cancer cell lines by the standard MTT [3-(4,5-dimethylthiazol-2-y1)-2,5-diphenytetrazolium bromide] method, and the results showed that $\mathbf{6}, \mathbf{1 0}$ and $\mathbf{1 1}$ exhibited moderate cytotoxicity against SMMC-7721, MCF-7 and SW480 cancer cell lines.
\end{abstract}

Keywords flavonoid; hesperidin; diosmetin; synthesis; biological activity

3'-O-甲基香叶木素(1)是我国南方药用植物 Striga asiatica (L.) O. Kuntze (Scrophulariaceae)的有效成分 ${ }^{[1]}$, 香叶木素-7- $O$ - 葡萄糖苷 (2) 是具有清热解毒等作用的蓬 子菜的主要化学成分之一 ${ }^{[2]}$, Lin 等 ${ }^{[3]}$ 在菊花中也发现了 香叶木素-7- $O$-葡萄糖苷(2)和香叶木素-7- $O$-半乳糖苷
(3)，而 3'- $O$-甲基香叶木素-7- $O$-葡萄糖苷(4)在埃及水苏 中首次被发现 ${ }^{[4]}$. 关于这些天然黄酮糖苷类化合物的生 物活性报道较少, 而关于香叶木素-7- $O$-半乳糖(3)及 3'- $O$-甲基香叶木素-7- $O$-葡萄糖苷(4)的化学合成更是未 见文献报道. 另外, 研究表明 $O$-法呢烯基化或 $O$-异戊

*E-mail: wangqiuan@yahoo.com

Received September 8, 2011; revised October 14, 2011; published online November 21, 2011.

Project supported by the Science and Technology Planning Project of Hunan Province (No. 2011FJ3214).

湖南省科技计划(No. 2011FJ3214)资助项目. 
烯基化可使黄酮类化合物的亲脂性得到提高, 有助于其 在生物体中透过细胞膜从而增强其生物活性 ${ }^{[5,6]}$.

为了进一步研究香叶木素黄酮类化合物的生物活 性, 探讨其构效关系, 我们以丰富易得的天然二氢黄酩 类橙皮苷(hesperdin, hespertin-7-rutinoside)为原料, 经脱 氢、甲基化(或苠基选择性保护酚羟基), 糖苷水解、相 转移催化下的糖苷化反应、异戊烯基化和法呢烯基化等 反应步骤, 分别合成了 $3^{\prime}-O$-甲基香叶木素(1), 香叶木 素-7- $O-\beta-D$ - 葡萄糖苷(2), 香叶木素-7- $O-\beta-D$-半乳糖苷 (3)，3'-O-甲基香叶木素-7- $O-\beta-D$-葡萄糖苷(4) 4 种天然
产物及 3'- $O$-甲基香叶木素-7- $O-\beta$ - $D$-半乳糖苷(5), 香叶 木素-7-O- $\beta-D$-乙酰葡萄糖苷(6), 香叶木素-7- $O-\beta-D$-乙 酰半乳糖苷(7), 3'- $O$-甲基香叶木素-7- $O-\beta-D$-乙酰葡萄 糖苷(8)，3'- $O$-甲基香叶木素-7- $O-\beta-D$-乙酰半乳糖苷(9), 7- $O$-异戊基香叶木素(10)，7- $O$-异戊烯基-3'- $O$-甲基香叶 木素(11)和 7- $O$-法呢烯基-'- $O$-甲基香叶木素(12) 8 种新 的香叶木素类衍生物. 并用 MTT 蛋白染色法对所合成 的目标化合物进行了体外抗肿瘤细胞生物活性测试. 系 列香叶木素类天然黄酮及其衍生物的合成路线见 Scheme 1.<smiles>[R20]c1cc(O)c2c(=O)cc(-c3ccc(OC)c(O)c3)oc2c1</smiles>

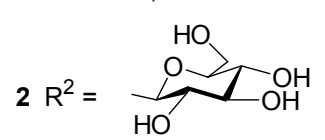

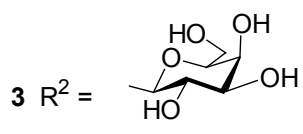<smiles>[R6]c1cc(O)c2c(c1)OC(c1ccc(OC)c(O)c1)CC2=O</smiles>

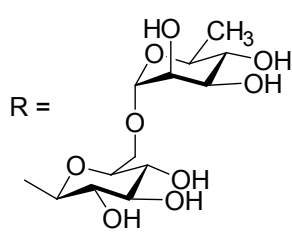
$\mathrm{R}^{1} \mathrm{O}$<smiles>COc1ccc(-c2cc(=O)c3c(O)cc(C)cc3o2)cc1</smiles>

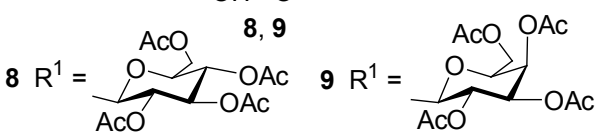<smiles>[R20]c1cc(O)c2c(=O)cc(-c3ccc(OC)c(OC)c3)oc2c1</smiles>

$\mathrm{RO}$

$5 \mathrm{R}^{2}=$<smiles>[R20]c1cc(O)c2c(=O)cc(-c3ccc(OC)c(O)c3)oc2c1</smiles>

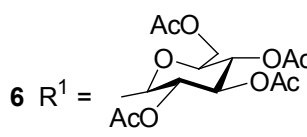

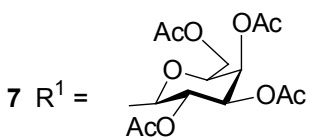<smiles>COc1cc(COc2cc(-c3cc(=O)c4c(O)cc(OCCC(C)C)cc4o3)ccc2OC)ccc1OCc1ccccc1</smiles><smiles>[R]c1cc(O)c2c(=O)cc(-c3ccc(OC)c(O)c3)oc2c1</smiles><smiles>COc1ccc(-c2cc(=O)c3c(O)cc(O)cc3o2)cc1O</smiles>
Diosmetin<smiles>[Y12]C=C1C=C(c2ccc(OC)c(OC)c2)Oc2cc([R9])cc(O)c21</smiles><smiles></smiles>

$12 \mathrm{R}^{3}=$<smiles>CC/C=C(\C)CC/C=C(\C)CCC=C(C)C</smiles><smiles>CC1OCC(O)C(O)C1O</smiles><smiles>[R]C#CC(O)[C@H](O)[C@H](O)[C@H](O)C(O)O</smiles><smiles>C1CCCC1</smiles>

Reagent and conditions: (a) $\mathrm{I}_{2}$, pyridine, $90 \sim 95{ }^{\circ} \mathrm{C}$; (b) $\mathrm{BnBr}, \mathrm{K}_{2} \mathrm{CO}_{3}$, DMF; (c) $\mathrm{H}^{+}, \mathrm{H}_{2} \mathrm{O}, \mathrm{C}_{2} \mathrm{H}_{5} \mathrm{OH}$; (d) $\left(\mathrm{CH}_{3}\right)_{2} \mathrm{SO}_{4}, 3 \% \mathrm{NaOH}^{\circ} 10 \sim 25{ }^{\circ} \mathrm{C}$; (e) acetylglycose bromide, $\mathrm{K}_{2} \mathrm{CO}_{3}, \mathrm{CHCl}_{3}, \mathrm{Bu}_{4} \mathrm{~N}^{+} \mathrm{Br}^{-}$, 45 $50{ }^{\circ} \mathrm{C}$; (f) $5 \% \mathrm{Pd} / \mathrm{C}, \mathrm{H}_{2}, \mathrm{CH}_{3} \mathrm{CO}_{2} \mathrm{C}_{2} \mathrm{H}_{5}, \mathrm{CH}_{3} \mathrm{OH}$, r.t.; (g) $\mathrm{CH}_{3} \mathrm{ONa}, \mathrm{CH}_{3} \mathrm{OH}$, reflux; (h) prenyl bromide or farnesyl bromide, $\mathrm{K}_{2} \mathrm{CO}_{3}$, dry acetone

Scheme 1 


\section{1 实验部分}

\section{1 试剂与仪器}

熔点在 XRC-1 型显微熔点仪上测定, 温度未校正; NMR 用 Bruker Am-400 型核磁共振仪测定(溶剂为 $\mathrm{CDCl}_{3}$ 或 DMSO- $d_{6}, \mathrm{TMS}$ 为内标); 质谱用 Agilent 1100 液一质联用仪或 ZAB-HS 型仪测定记录; 红外光谱用 FD-5DX 仪( $\mathrm{KBr}$ 压片)测定; 元素分析用德国元素分析 仪(Vario ELL III)测定; 柱层析用硅胶(青岛海洋化工厂 产品). 所用试剂和溶剂为化学纯和分析纯; 要求无水 的溶剂均经除水和重蒸处理.

地奥明(Diosmin)和香叶木素(Diosmetin)按文献[7] 以橙皮苷为原料分别经脱氢、糖苷水解制备; 溴代2,3,4,6-四- $O$-乙酰基- $\alpha$ - $D$-吡喃葡萄糖和溴代- $2,3,4,6$-四$O$-乙酰基- $\alpha$ - $D$-吡喃半乳糖参考文献[8]制备.

\subsection{3'-O-苄基香叶木素(13)的合成}

在 $100 \mathrm{~mL}$ 三口瓶中加入地奥明(2.215 g, 3.64 $\mathrm{mmol})$, 无水 $\mathrm{K}_{2} \mathrm{CO}_{3}(4 \mathrm{~g}, 29 \mathrm{mmol}), \mathrm{N}_{2}$ 保护下, 加入无 水 DMF $23 \mathrm{~mL}$, 室温摚拌 $1 \mathrm{~h}$, 再缓慢滴加 $\mathrm{BnBr}(0.5$ $\mathrm{mL}, 4.19 \mathrm{mmol}$ ), 油浴升温至 $95{ }^{\circ} \mathrm{C}$ 左右搅拌反应 $4 \mathrm{~h}$, 将 反应液倒入冰水中, 有黄色固体析出, 减压抽滤, 水洗, 得固体. 将此固体转移到 $250 \mathrm{~mL}$ 单口瓶中, 加入 120 $\mathrm{mL}$ 无水乙醇, 室温搅拌下滴加 $10 \mathrm{~mL}$ 浓 $\mathrm{HCl}$, 回流摚拌 $7 \mathrm{~h}$, 反应液冷却至室温, 减压蒸除部分乙醇, 将剩下的 乙醇溶液倒入水中, 有淡黄色固体析出, 减压抽滤, 滤 饼水洗, 干燥, 硅胶柱层析分离[洗脱剂: $V$ (石油醚)： $V($ 乙酸乙酯 $)=2: 1$ ], 得黄色粉末状固体 $0.68 \mathrm{~g}$, 产率 $56 \%$. m.p. $230 \sim 232{ }^{\circ} \mathrm{C} ;{ }^{1} \mathrm{H}$ NMR (DMSO- $d_{6}, 400 \mathrm{MHz}$ ) $\delta: 12.93$ (s, 1H, 5-OH), 10.88 (s, 1H, 7-OH), 7.68 7.73 (m, 2H, 2",6"-H), 7.36 7.51 (m, 5H, 2',6',3",4",5"-H), 7.16 (d, $\left.J=8.8 \mathrm{~Hz}, 1 \mathrm{H}, 5^{\prime}-\mathrm{H}\right), 6.97$ (s, 1H, 3-H), 6.53 (s, $1 \mathrm{H}, 6-\mathrm{H}), 6.21(\mathrm{~d}, J=2.0 \mathrm{~Hz}, 1 \mathrm{H}, 8-\mathrm{H}), 5.24(\mathrm{~s}, 2 \mathrm{H}$, $\left.3^{\prime}-\mathrm{OCH}_{2}\right), 3.87$ (s, 3H, 4'--OCH 3 ); MS $\left(\mathrm{FAB}^{+}\right) \mathrm{m} / z$ : 391 $[\mathrm{M}+1]^{+}$.

\subsection{3'-O-甲基香叶木素(1)的合成}

称取地奥明 $(3.088 \mathrm{~g}, 5.07 \mathrm{mmol})$ 加入到 $250 \mathrm{~mL}$ 三 口瓶中, 再加入 $3 \% \mathrm{NaOH}$ 溶液 $100 \mathrm{~mL}$, 控制温度在 $10 \sim 25{ }^{\circ} \mathrm{C}$ 搅拌 $30 \mathrm{~min}$, 分次缓慢滴加 $\left(\mathrm{CH}_{3}\right)_{2} \mathrm{SO}_{4}(6.8$ $\mathrm{mL}, 71.35 \mathrm{mmol}$ ), 摚拌反应 $8 \mathrm{~h}$, 反应过程中控制 $\mathrm{pH}$ 为 碱性, 若反应体系变成酸性需要及时补加 $3 \% \mathrm{NaOH}$ 溶 液. 反应结束, 将反应液调制酸性, 过滤析出的固体, 将滤液加入到 $250 \mathrm{~mL}$ 单口瓶中, 摚拌下滴加 $6 \mathrm{~mL}$ 浓 $\mathrm{H}_{2} \mathrm{SO}_{4}$, 油浴升温, 回流搅拌 $6 \mathrm{~h}$, 反应过程中有黄色固 体析出. 反应结束后, 将反应液冷却至室温, 减压抽滤, 滤液用乙酸乙酯萃取, 滤饼水洗至中性, 干燥, 硅胶柱
层析分离 [洗脱剂: $V$ (石油醚) $: V($ 乙酸乙酯 $)=2: 1$ ], 得 黄色粉末状固体 $0.72 \mathrm{~g}$, 产率 $45 \%$. m.p. $278 \sim 280{ }^{\circ} \mathrm{C}$ [文 献值 ${ }^{[1]}$ : 276 $\left.278{ }^{\circ} \mathrm{C}\right] ;{ }^{1} \mathrm{H}$ NMR $\left(\mathrm{CDCl}_{3}, 400 \mathrm{MHz}\right) \delta$ : 12.93 (s, 1H, 5-OH), 10.85 (s, 1H, 7-OH), 7.69 (dd, $J=$ $\left.8.2,2.0 \mathrm{~Hz}, 1 \mathrm{H}, 6{ }^{\prime}-\mathrm{H}\right), 7.57$ (d, $\left.J=2.0 \mathrm{~Hz}, 1 \mathrm{H}, 2^{\prime}-\mathrm{H}\right), 7.13$ (d, $\left.J=8.2 \mathrm{~Hz}, 1 \mathrm{H}, 5^{\prime}-\mathrm{H}\right), 6.98$ (s, 1H, 3-H), 6.54 (d, $J=2.0$ $\mathrm{Hz}, 1 \mathrm{H}, 6-\mathrm{H}), 6.21$ (d, $J=2.0 \mathrm{~Hz}, 1 \mathrm{H}, 8-\mathrm{H}), 3.88,3.86$ (each s, each $\left.3 \mathrm{H}, 4^{\prime}-\mathrm{OCH}_{3}, 3^{\prime}-\mathrm{OCH}_{3}\right)$; IR ( $\left.\mathrm{KBr}\right) v: 3100$, $1650,1615,1570,1510,1160,1015,850,765 \mathrm{~cm}^{-1}$; MS $\left(\mathrm{FAB}^{+}\right) \mathrm{m} / z: 315[\mathrm{M}+1]^{+}$.

\section{4 香叶木素-7-O- $\beta$ - $D$-乙酰葡萄糖苷(6)的合成}

向 $100 \mathrm{~mL}$ 单口瓶中, 加入 $\mathbf{1 3}(200 \mathrm{mg}, 0.51 \mathrm{mmol})$, $50 \mathrm{mg} \mathrm{Bu} 4 \mathrm{~N}^{+} \mathrm{Br}^{-}$(TBAB), 再分别加入 $20 \mathrm{~mL} 0.25$ $\mathrm{mol} / \mathrm{L}$ 的 $\mathrm{K}_{2} \mathrm{CO}_{3}$ 溶液和 $20 \mathrm{~mL} \mathrm{CHCl}_{3}$, 室温下摚拌 $1 \mathrm{~h}$ 后, 向体系中加入溴代-2,3,4,6-四- $O$-乙酰基- $\alpha$ - $D$-吡喃葡萄 糖 $(0.42 \mathrm{~g}, 1.02 \mathrm{mmol})$, 升温至 $45 \sim 50{ }^{\circ} \mathrm{C}$, 反应过夜. 反 应结束后, 分离得到有机相, 水相再用 $\mathrm{CH}_{2} \mathrm{Cl}_{2}$ 萃取三 次, 合并有机相, 无水硫酸钠干燥, 过滤, 蒸除溶剂得 粗品. 硅胶柱层析分离[洗脱剂： $V$ (石油醚)： $V$ (乙酸乙 酯 $)=2: 1$ ], 得油状物, 用甲醇重结晶, 得到黄色固体 3'- $O$-苄基香叶木素-7- $O-\beta$ - $D$-乙酰葡萄糖苷 $212 \mathrm{mg}$, 产 率 58.9\%. m.p. $105 \sim 107{ }^{\circ} \mathrm{C} ;{ }^{1} \mathrm{H}$ NMR $\left(\mathrm{CDCl}_{3}, 400 \mathrm{MHz}\right)$ $\delta: 12.82$ (s, 1H, 5-OH), $7.49 \sim 7.53$ (m, 3H, 2"',6',6"'-H), $7.32 \sim 7.42$ (m, 4H, 2',3"',4"',5"'-H), 7.00 (d, $J=8.8 \mathrm{~Hz}$, 1H, 5'-H), 6.52 (s, 1H, 3-H), 6.55 (d, $J=2.0 \mathrm{~Hz}, 1 \mathrm{H}, 6-\mathrm{H})$, $6.45(\mathrm{~d}, J=2.4 \mathrm{~Hz}, 1 \mathrm{H}, 8-\mathrm{H}), 5.28 \sim 5.33\left(\mathrm{~m}, 2 \mathrm{H}, 6 "-\mathrm{CH}_{2}\right)$, 5.23 (s, 2H, 3'-OCH $), 5.19$ (d, J=7.6 Hz, 1H, 2"-H), 5.17 (d, $J=9.6,1 \mathrm{H}, 1 "-\mathrm{H}), 4.30(\mathrm{dd}, J=12.0,6.0 \mathrm{~Hz}, 1 \mathrm{H}$, 3"-H), 4.21 (dd, $J=12.4,2.4 \mathrm{~Hz}, 1 \mathrm{H}, 4 "-\mathrm{H}), 3.97$ (s, 3H, 4'-OCH ${ }_{3}$ ), 3.93 3.94 (m, 1H, 5"-H), 2.10, 2.08, 2.07, 2.05 (each s, each $3 \mathrm{H}, \mathrm{COCH}_{3}$ ).

在 $100 \mathrm{~mL}$ 放有磁子的三口瓶加入 $253 \mathrm{mg} 5 \% \mathrm{Pd} / \mathrm{C}$, 将其充满氢气，然后将 3 '- $O$-芐基香叶木素-7- $O-\beta-D-$ 乙 酰葡萄糖苷 $(200 \mathrm{mg}, 0.28 \mathrm{mmol})$ 用 $20 \mathrm{~mL}$ 乙酸乙酯和 10 $\mathrm{mL}$ 甲醇的混合溶液溶解, 用注射器加入三口瓶中, 室 温氢解反应 $12 \mathrm{~h}$. 反应结束后, 过滤除去 $\mathrm{Pd} / \mathrm{C}$, 用乙酸 乙酯、甲醇交替淋洗 $\mathrm{Pd} / \mathrm{C}$, 滤液减压蒸干得到粗产物, 硅胶柱层析分离 [洗脱剂: $V$ (石油醚) $: V$ (乙酸乙酯 $)=$ 3:2], 得黄色固体 $160 \mathrm{mg}$, 产率 $91.5 \%$. m.p. 221 223 ${ }^{\circ} \mathrm{C} ;{ }^{1} \mathrm{H}$ NMR $\left(\mathrm{CDCl}_{3}, 400 \mathrm{MHz}\right) \delta$ : 12.81 (s, $\left.1 \mathrm{H}, 5-\mathrm{OH}\right)$, 7.45 (dd, $\left.J=8.4,2.0 \mathrm{~Hz}, 1 \mathrm{H}, 66^{\prime}-\mathrm{H}\right), 7.42(\mathrm{~d}, J=2.0 \mathrm{~Hz}$, $1 \mathrm{H}, 2-\mathrm{H}), 6.97$ (d, $J=8.4 \mathrm{~Hz}, 1 \mathrm{H}, 5$ '-H), 6.59 (s, 1H, 3-H), $6.58(\mathrm{~d}, J=2.0 \mathrm{~Hz}, 1 \mathrm{H}, 6-\mathrm{H}), 6.44(\mathrm{~d}, J=2.4 \mathrm{~Hz}, 1 \mathrm{H}$, $8-\mathrm{CH}_{2}$ ), $5.76\left(\mathrm{~s}, 1 \mathrm{H}, 3^{\prime}-\mathrm{OH}\right), 5.30 \sim 5.33(\mathrm{~m}, 2 \mathrm{H}, 6 "-\mathrm{H})$, $5.17 \sim 5.19$ (m, 1H, 2"-H), 5.16 (d, $J=9.2 \mathrm{~Hz}, 1 \mathrm{H}, 1 "-\mathrm{H})$, 
4.30 (dd, $J=12.4,4.0 \mathrm{~Hz}, 1 \mathrm{H}, 3 "-\mathrm{H}), 4.21$ (dd, $J=12.0$, $\left.2.4 \mathrm{~Hz}, 1 \mathrm{H}, 4^{\prime \prime}-\mathrm{H}\right), 3.99$ (s, 3H, 4'-OCH ${ }_{3}$ ), 3.93 3.97 (m, $1 \mathrm{H}, 5 "-\mathrm{H}$ ), 2.12, 2.08, 2.07, 2.05 (each s, each $3 \mathrm{H}$, $\mathrm{COCH}_{3}$ ); MS (ESI) $m / z: 653[\mathrm{M}+\mathrm{Na}]^{+}$. Anal. calcd for $\mathrm{C}_{30} \mathrm{H}_{30} \mathrm{O}_{15}$ : C 57.14, H 4.80; found C 57.29, H 4.71 .

\section{5 香叶木素-7-O- $\beta-D$-葡萄糖苷(2)的合成}

在 $100 \mathrm{~mL}$ 单口烧瓶中, 加入 $\mathbf{5}(49 \mathrm{mg}, 0.078 \mathrm{mmol})$, 用 $15 \mathrm{~mL}$ 甲醇加热搅拌至溶解, 此时再加入 $5 \mathrm{~mL}$ 甲醇 钠溶液 ( $5 \mathrm{~mL}$ 甲醇和 $4.3 \mathrm{mg}$ 钠配置而成), 回流摚拌反应 $3 \mathrm{~h}$ 后, TLC 跟踪反应至原料点消失, 停止反应, 冷却至 室温, 减压蒸除溶剂, 硅胶柱层析分离[洗脱剂: 用乙酸 乙酯及乙酸乙酯和乙醇的混合溶剂进行梯度洗脱], 得 到淡黄色固体 $17 \mathrm{mg}$, 产率 47.1\%. m.p. 259 261 ${ }^{\circ} \mathrm{C} ;{ }^{1} \mathrm{H}$ NMR (DMSO- $\left.d_{6}, 400 \mathrm{MHz}\right) \delta$ : 12.94 (s, 1H, 5-OH), 9.47 (s, 1H, 3'-OH), 7.59 (dd, $\left.J=8.6,2.4 \mathrm{~Hz}, 1 \mathrm{H}, 66^{\prime}-\mathrm{H}\right), 7.46$ (d, $\left.J=2.4 \mathrm{~Hz}, 1 \mathrm{H}, 2^{\prime}-\mathrm{H}\right), 7.12$ (d, $\left.J=8.8 \mathrm{~Hz}, 1 \mathrm{H}, 5^{\prime}-\mathrm{H}\right), 6.84$ (s, 1H, 3-H), 6.82 (d, $J=2.0 \mathrm{~Hz}, 1 \mathrm{H}, 6-\mathrm{H}), 6.46$ (d, $J=2.0$ $\mathrm{Hz}, 1 \mathrm{H}, 8-\mathrm{H}), 5.41$ (d, $\left.J=4.8 \mathrm{~Hz}, 1 \mathrm{H}, 2^{\prime \prime}-\mathrm{OH}\right), 5.14$ (d, $J=$ $4.8 \mathrm{~Hz}, 1 \mathrm{H}, 3 "-\mathrm{OH}), 5.09$ (d, $J=9.0 \mathrm{~Hz}, 1 \mathrm{H}, 1 "-\mathrm{H}), 5.07$ (br s, 1H, 4"-OH), 4.62 (t, $J=5.6 \mathrm{~Hz}, 1 \mathrm{H}, 6 "-\mathrm{OH}$ ), 3.88 (s, $\left.3 \mathrm{H}, 4{ }^{\prime}-\mathrm{OCH}_{3}\right), 3.71$ (dd, $\left.J=10.2,5.2 \mathrm{~Hz}, 1 \mathrm{H}, 3 "-\mathrm{H}\right)$, $3.45 \sim 3.49$ (m, 2H, 6"-- $\left.\mathrm{CH}_{2}\right), 3.17 \sim 3.31$ (m, 3H, 2", $4 ", 5 "-\mathrm{H})$; MS (ESI) $m / z: 485[\mathrm{M}+\mathrm{Na}]^{+}$.

\section{6 香叶木素-7-O- $\beta-D$-乙酰半乳糖苷(7)的合成}

7 的合成方法同 6, 以 13 和澳代-2,3,4,6-四- $O$-乙酰 基- $\alpha$ - $D$-吡喃半乳糖为原料, 得黄色固体 $3{ }^{\prime}-O$-芐基香叶 木素-7-O- $\beta$-D-乙酰半乳糖苷, 产率 51\%. m.p. 116 119 ${ }^{\circ} \mathrm{C} ;{ }^{1} \mathrm{H}$ NMR $\left(\mathrm{CDCl}_{3}, 400 \mathrm{MHz}\right) \delta: 7.48 \sim 7.53(\mathrm{~m}, 3 \mathrm{H}$, 2"',6',6"'-H), 7.34 7.41 (m, 3H, 2',3"',4"',5"'-H), 7.01 (d, $\left.J=8.4 \mathrm{~Hz}, 1 \mathrm{H}, 5{ }^{\prime}-\mathrm{H}\right), 6.52(\mathrm{~s}, 1 \mathrm{H}, 3-\mathrm{H}), 6.56(\mathrm{~d}, J=1.2$ $\mathrm{Hz}, 1 \mathrm{H}, 6-\mathrm{H}), 6.46$ (d, $J=1.2 \mathrm{~Hz}, 1 \mathrm{H}, 8-\mathrm{H}), 5.48 \sim 5.53$ (m, 3H, 2",5"-H), $5.23\left(\mathrm{~s}, 2 \mathrm{H}, 3\right.$ '- $\left.\mathrm{OCH}_{2}\right), 5.13 \sim 5.16(\mathrm{~m}$, $3 \mathrm{H}, 1 ", 3 "-\mathrm{H}), 4.25$ (dd, $J=11.2,8.8 \mathrm{~Hz}, 1 \mathrm{H}, 4 "-\mathrm{H}), 4.14 \sim$ 4.20 (m, 2H, 6"- $\mathrm{CH}_{2}$ ), 3.97 (s, 3H, 4'-- $\mathrm{OCH}_{3}$ ), 2.20, 2.17, 2.09, 2.03 (each s, each $3 \mathrm{H}, \mathrm{COCH}_{3}$ ). 然后将 3'- $\mathrm{O}$-芐基香 叶木素-7- $O-\beta-\mathrm{D}$-乙酰半乳糖苷室温催化氢化, 硅胶柱 层析分离[洗脱剂: $V$ (石油醚) $: V($ 乙酸乙酯 $)=3: 2$ ], 得 黄色固体 7, 产率 90.2\%. m.p. $163 \sim 166{ }^{\circ} \mathrm{C} ;{ }^{1} \mathrm{H}$ NMR $\left(\mathrm{CDCl}_{3}, 400 \mathrm{MHz}\right) \delta: 12.80(\mathrm{~s}, 1 \mathrm{H}, 5-\mathrm{OH}), 7.542 \sim 7.45$ (m, 2H, 2',6'-H), 6.97 (d, J=8.4 Hz, 1H, 5'-H), 6.59 (s, 1H, 3-H), 6.60 (d, $J=2.4 \mathrm{~Hz}, 1 \mathrm{H}, 6-\mathrm{H}), 6.45(\mathrm{~d}, J=2.0 \mathrm{~Hz}$, $1 \mathrm{H}, 8-\mathrm{H}), 5.86\left(\mathrm{~s}, 1 \mathrm{H}, 3^{\prime}-\mathrm{OH}\right), 5.49 \sim 5.55(\mathrm{~m}, 3 \mathrm{H}$, 2",5"-H), $5.13 \sim 5.16$ (m, 3H, 1",3"-H), 4.21 4.23 (m, 2H, 6"-- $\mathrm{CH}_{2}$ ), $4.15 \sim 4.17$ (m, 1H, 4"-H), 3.98 (s, 3H, 4'-- $\mathrm{OCH}_{3}$ ), 2.20, 2.11, 2.09, 2.03 (each s, each 3H, $\mathrm{COCH}_{3}$ ); MS (ESI) $m / z: 653[\mathrm{M}+\mathrm{Na}]^{+}$. Anal. calcd for $\mathrm{C}_{30} \mathrm{H}_{30} \mathrm{O}_{15}: \mathrm{C} 57.14, \mathrm{H}$ 4.80; found C 57.28, H 4.65

\section{7 香叶木素-7-O- $\beta-D$-半乳糖苷(3)的合成}

3 的合成方法同 2 , 以 7 为原料, 得淡黄色固体, 产 率 45\%. m.p. $250 \sim 252{ }^{\circ} \mathrm{C}$; ${ }^{1} \mathrm{H}$ NMR (DMSO-d $d_{6}, 400$ MHz) $\delta: 12.95$ (s, 1H, 5-OH), 9.49 (s, 1H, 3'-OH), 7.58 (d, $\left.J=8.8 \mathrm{~Hz}, 1 \mathrm{H}, 6^{\prime}-\mathrm{H}\right), 7.46$ (d, $\left.J=1.6 \mathrm{~Hz}, 1 \mathrm{H}, 2^{\prime}-\mathrm{H}\right), 7.11$ (d, $\left.J=8.8 \mathrm{~Hz}, 1 \mathrm{H}, 5^{\prime}-\mathrm{H}\right), 6.81 \sim 6.84(\mathrm{~m}, 2 \mathrm{H}, 3,6-\mathrm{H}), 6.46$ (s, $1 \mathrm{H}, 8-\mathrm{H}), 5.26$ (d, $J=4.8 \mathrm{~Hz}, 1 \mathrm{H}, 2$ "-OH), 5.04 (d, $J=$ $7.2 \mathrm{~Hz}, 1 \mathrm{H}, 1 "-\mathrm{H}$ ), 4.93 (d, $\left.J=5.6 \mathrm{~Hz}, 1 \mathrm{H}, 4^{\prime \prime}-\mathrm{OH}\right), 4.73$ (t, $J=5.2 \mathrm{~Hz}, 1 \mathrm{H}, 6 "-\mathrm{OH}), 4.58$ (d, $J=4.8 \mathrm{~Hz}, 1 \mathrm{H}, 3 "-\mathrm{OH})$, $3.88\left(\mathrm{~s}, 3 \mathrm{H}, 4^{\prime}-\mathrm{OCH}_{3}\right), 3.56 \sim 3.73(\mathrm{~m}, 6 \mathrm{H}$, sugar-H); $\mathrm{MS}$ (ESI) $m / z: 485[\mathrm{M}+\mathrm{Na}]^{+}$.

\subsection{3 '-O-甲基香叶木素-7-O- $\beta$ - $D$-乙酰葡萄糖苷(8)的 合成}

8 的合成方法同 6 , 以 1 和溴代-2,3,4,6-四- $O$-乙酰 基- $\alpha$-D-吡喃葡萄糖为原料, 得黄色固体, 产率 $62.1 \%$. m.p. $120 \sim 122{ }^{\circ} \mathrm{C} ;{ }^{1} \mathrm{H}$ NMR $\left(\mathrm{CDCl}_{3}, 400 \mathrm{MHz}\right) \delta: 7.53$ (dd, $\left.J=8.4,2.0 \mathrm{~Hz}, 1 \mathrm{H}, 6{ }^{\prime}-\mathrm{H}\right), 7.33(\mathrm{~d}, J=2.0 \mathrm{~Hz}, 1 \mathrm{H}$, 2'-H), 7.00 (d, $\left.J=8.8 \mathrm{~Hz}, 1 \mathrm{H}, 5^{\prime}-\mathrm{H}\right), 6.62(\mathrm{~s}, 1 \mathrm{H}, 3-\mathrm{H})$, $6.60(\mathrm{~d}, J=2.0 \mathrm{~Hz}, 1 \mathrm{H}, 6-\mathrm{H}), 6.45(\mathrm{~d}, J=2.0 \mathrm{~Hz}, 1 \mathrm{H}$, 8-H), $5.31 \sim 5.34$ (m, 2H, 6"-CH $), 5.21(\mathrm{~d}, J=6.8 \mathrm{~Hz}, 1 \mathrm{H}$, 2"-H), 5.17 (d, $J=9.2 \mathrm{~Hz}, 1 \mathrm{H}, 1 "-\mathrm{H}), 4.31$ (dd, $J=12.4$, $6.0 \mathrm{~Hz}, 1 \mathrm{H}, 3 "-\mathrm{H}), 4.21$ (dd, $J=12.4,5.0 \mathrm{~Hz}, 1 \mathrm{H}, 4 "-\mathrm{H}$ ), $3.99,3.98$ (each s, each $\left.3 \mathrm{H}, 4^{\prime}-\mathrm{OCH}_{3}, 3^{\prime}-\mathrm{OCH}_{3}\right), 3.93$ (m, $1 \mathrm{H}, 5 "-\mathrm{H}$ ), 2.11, 2.08, 2.07, 2.05 (each s, each 3H, $\mathrm{COCH}_{3}$ ); $\mathrm{MS}$ (ESI) $m / z: 667[\mathrm{M}+\mathrm{Na}]^{+}$. Anal. calcd for $\mathrm{C}_{31} \mathrm{H}_{32} \mathrm{O}_{15}$ : C 57.76, H 5.00; found C 57.56, H 4.91.

\subsection{3 -O-甲基香叶木素-7-O- $\beta-D$-葡萄糖苷(4)的合成}

4 的合成方法同 2 , 以 8 为原料, 得淡黄色固体, 产 率 $42 \%$. m.p. $234 \sim 236{ }^{\circ} \mathrm{C}$; ${ }^{1} \mathrm{H}$ NMR (DMSO- $d_{6}, 400$ MHz) $\delta: 12.92(\mathrm{~s}, 1 \mathrm{H}, 5-\mathrm{OH}), 7.59$ (d, $J=7.6 \mathrm{~Hz}, 1 \mathrm{H}$, 6'-H), 7.46 (s, 1H, 2'-H), 7.12 (d, $\left.J=8.8 \mathrm{~Hz}, 1 \mathrm{H}, 5^{\prime}-\mathrm{H}\right)$, 7.08 (s, 1H, 3-H), 6.89 (s, 1H, 6-H), 6.46 (s, 1H, 8-H), 5.42 (br, 1H, 2"-OH), 5.17 (br, 1H, 3"-OH), 5.09 (br, 1H, 4"-OH), 5.07 (d, J=7.2 Hz, 1H, 1"-H), 4.65 (br, 1H, 6"-OH), 3.86, 3.88 (each s, each $3 \mathrm{H}, 3^{\prime}-\mathrm{OCH}_{3}, 4^{\prime}-\mathrm{OCH}_{3}$ ), $3.70 \sim 3.72(\mathrm{~m}, 1 \mathrm{H}, 3 "-\mathrm{H}), 3.44 \sim 3.48\left(\mathrm{~m}, 2 \mathrm{H}, 6 "-\mathrm{CH}_{2}\right)$, $3.17 \sim 3.31$ (m, 3H, 2",4",5"-H); MS (ESI) m/z: 499 [M+ $\mathrm{Na}]^{+}$.

1.103 '-O-甲基香叶木素-7-O- $\beta$ - $D$-乙酰半乳糖苷(9)的 合成

9 的合成方法同 6, 以 1 和溴代-2,3,4,6-四- $O$-乙酰 基- $\alpha$ - $D$-吡喃半乳糖为原料, 得黄色固体 $\mathbf{9}$, 产率 $55 \%$. 
m.p. $118 \sim 120{ }^{\circ} \mathrm{C} ;{ }^{1} \mathrm{H}$ NMR $\left(\mathrm{CDCl}_{3}, 400 \mathrm{MHz}\right) \delta: 12.82$ (s, 1H, 5-OH), 7.54 (dd, $\left.J=8.4,2.0 \mathrm{~Hz}, 1 \mathrm{H}, 66^{\prime}-\mathrm{H}\right), 7.34$ (d, $\left.J=2.0 \mathrm{~Hz}, 1 \mathrm{H}, 2^{\prime}-\mathrm{H}\right), 7.00$ (d, $\left.J=8.8 \mathrm{~Hz}, 1 \mathrm{H}, 5^{\prime}-\mathrm{H}\right), 6.62$ (s, 1H, 3-H), 6.61 (d, $J=2.4 \mathrm{~Hz}, 1 \mathrm{H}, 6-\mathrm{H}), 6.45$ (d, $J=2.0$ $\mathrm{Hz}, 1 \mathrm{H}, 8-\mathrm{H}), 5.48 \sim 5.54$ (m, 3H, 2",5"-H), 5.12 $\sim 5.17$ (m, 3H, 1",3"-H), 4.25 (dd, $J=11.2,7.2 \mathrm{~Hz}, 1 \mathrm{H}, 4 "-\mathrm{H}$ ), $4.14 \sim 4.20$ (m, 2H, 6"-- $\mathrm{CH}_{2}$ ), 3.99, 3.97 (each s, each 3H, 4'- $-\mathrm{OCH}_{3}, 3$ '- $-\mathrm{OCH}_{3}$ ), 2.20, 2.10, 2.09, 2.03 (each s, each $\left.3 \mathrm{H}, \mathrm{COCH}_{3}\right)$. MS (ESI) $m / z: 667[\mathrm{M}+\mathrm{Na}]^{+}$. Anal. calcd for $\mathrm{C}_{31} \mathrm{H}_{32} \mathrm{O}_{15}$ : C 57.76, H 5.00; found C 57.57, H 4.93.

\subsection{3 '-O-甲基香叶木素-7-O- $\beta$ - $D$-半乳糖苷(5)的合成}

5 的合成方法同 2 , 以 9 为原料, 得黄色固体, 产率 $40.1 \%$. m.p. $232 \sim 235{ }^{\circ} \mathrm{C} ;{ }^{1} \mathrm{H}$ NMR (DMSO- $d_{6}, 400 \mathrm{MHz}$ ) $\delta: 12.90$ (s, 1H, 5-OH), 7.69 (dd, $\left.J=8.4,2.0 \mathrm{~Hz}, 1 \mathrm{H}, 6{ }^{\prime}-\mathrm{H}\right)$, $7.56\left(\mathrm{~d}, J=1.6 \mathrm{~Hz}, 1 \mathrm{H}, 2^{\prime}-\mathrm{H}\right), 7.11(\mathrm{~d}, J=8.8 \mathrm{~Hz}, 1 \mathrm{H}$, 5'-H), 7.04 (s, 1H, 3-H), 6.85 (d, J=2.0 Hz, 1H, 6-H), 6.43 (d, $J=2.0 \mathrm{~Hz}, 1 \mathrm{H}, 8-\mathrm{H}), 5.28$ (d, $J=4.8 \mathrm{~Hz}, 1 \mathrm{H}, 2 "-\mathrm{OH})$, 4.99 (d, $\left.J=7.6 \mathrm{~Hz}, 2 \mathrm{H}, 1 "-\mathrm{H}, 4^{\prime \prime}-\mathrm{OH}\right), 4.73$ (t, $J=5.2 \mathrm{~Hz}$, $1 \mathrm{H}, 6 "-\mathrm{OH}), 4.58$ (d, J=4.0 Hz, 1H, 3"-OH), 3.85, 3.82 (each s, each 3H, 3'- $\left.\mathrm{OCH}_{3}, 4^{\prime}-\mathrm{OCH}_{3}\right), 3.38 \sim 3.67(\mathrm{~m}, 6 \mathrm{H}$, sugar-H); IR (KBr) v: 3413, 3228, 2169, 2042, 1660, 1615, 1400, 1257, $897 \mathrm{~cm}^{-1}$; MS (ESI) $m / z: 499[\mathrm{M}+\mathrm{Na}]^{+}$. Anal. calcd for $\mathrm{C}_{23} \mathrm{H}_{24} \mathrm{O}_{11}$ : C 57.98, $\mathrm{H}$ 5.08; found $\mathrm{C}$ 58.18, H 5.21 .

\subsection{7-O-异戊基香叶木素(10)的合成}

称取 $13(70 \mathrm{mg}, 0.18 \mathrm{mmol})$, 无水 $\mathrm{K}_{2} \mathrm{CO}_{3}(127 \mathrm{mg}$, $0.92 \mathrm{mmol}$ )加入到 $50 \mathrm{~mL}$ 单口瓶中, 再加入 $10 \mathrm{~mL}$ 无水 丙酮, $50{ }^{\circ} \mathrm{C}$ 左右搅拌 $1 \mathrm{~h}$, 缓慢滴加法呢烯基溴 $(0.03 \mathrm{ml}$, $0.26 \mathrm{mmol}$ )与 $2 \mathrm{~mL}$ 丙酮的混合溶液, TLC 跟踪反应至原 料点消失, 约 $2 \mathrm{~h}$, 停止反应, 冷却至室温, 过滤, 用少 量丙酮润洗滤渣, 滤液旋干得粗产物. 硅胶柱层析分离 [洗脱剂: $V$ (石油醚) : $V$ (乙酸乙酯 $)=10: 1$ ], 得黄色固 体 7- $O$-异戊烯基-3'-O-苠基香叶木素 $77 \mathrm{mg}$, 产率 93\%. m.p. $130 \sim 132{ }^{\circ} \mathrm{C} ;{ }^{1} \mathrm{H}$ NMR $\left(\mathrm{CDCl}_{3}, 400 \mathrm{MHz}\right) \delta: 12.77$ (s, $1 \mathrm{H}, 5-\mathrm{OH}), 7.48 \sim 7.52$ (m, 3H, 2"',4"',6"'-H), 7.38 7.42 (m, 3H, 2',3"',5"'-H), 7.33 (dd, $J=8.4,2.0 \mathrm{~Hz}, 1 \mathrm{H}$, 6'-H), 6.99 (d, $\left.J=8.4 \mathrm{~Hz}, 1 \mathrm{H}, 5^{\prime}-\mathrm{H}\right), 6.49$ (s, 1H, 3-H), $6.45(\mathrm{~d}, J=2.0 \mathrm{~Hz}, 1 \mathrm{H}, 6-\mathrm{H}), 6.37(\mathrm{~d}, J=2.0 \mathrm{~Hz}, 1 \mathrm{H}$, $8-\mathrm{H}), 5.48 \sim 5.52(\mathrm{~m}, 1 \mathrm{H}, 2 \mathrm{\prime}-\mathrm{H}), 5.23$ (s, $\left.2 \mathrm{H}, 3^{\prime}-\mathrm{OCH}_{2}\right)$, 4.59 (d, $J=6.4 \mathrm{~Hz}, 1 \mathrm{H}, 1$ '- $\left.\mathrm{CH}_{2}\right), 3.97$ (s, 3H, 4'- $-\mathrm{OCH}_{3}$ ), 1.82, 1.78 (each s, each 3H, 4", 5"-- $\mathrm{CH}_{3}$ ).

室温氢解同 6, 硅胶柱层析分离[洗脱剂: $V($ 石油 醚) $: V($ 乙酸乙酯 $)=4: 1$ ], 得黄色固体 $52 \mathrm{mg}$, 产率 $87.8 \%$. m.p. $138 \sim 141^{\circ} \mathrm{C} ;{ }^{1} \mathrm{H}$ NMR $\left(\mathrm{CDCl}_{3}, 400 \mathrm{MHz}\right) \delta$ : 12.76 (s, 1H, 5-OH), 7.46 (dd, $J=8.4,2.0 \mathrm{~Hz}, 1 \mathrm{H}, 6$ '- $\mathrm{H}$ ), $7.43\left(\mathrm{~d}, J=2.0 \mathrm{~Hz}, 1 \mathrm{H}, 2^{\prime}-\mathrm{H}\right), 6.96(\mathrm{~d}, J=8.4 \mathrm{~Hz}, 1 \mathrm{H}$, 5'-H), 6.56 (s, 1H, 3-H), 6.48 (d, J=2.4 Hz, 1H, 6-H), 6.35 (d, $J=2.0 \mathrm{~Hz}, 1 \mathrm{H}, 8-\mathrm{H}), 5.75\left(\mathrm{~s}, 1 \mathrm{H}, 3^{\prime}-\mathrm{OH}\right), 4.06$ (t, $J=$ $6.8 \mathrm{~Hz}, 1 \mathrm{H}, 1$ "-H), 3.98 (s, 3H, 4'-OCH 3$), 1.84 \sim 1.86(\mathrm{~m}$, $1 \mathrm{H}, 3$ "-H), $1.69 \sim 1.74$ (m, 2H, 2"-CH $), 0.99,0.97$ (each s, each $3 \mathrm{H}, 4 ", 5 "-\mathrm{CH}_{3}$ ); IR (KBr) v: 3413, 3228, 2169, 2030, 1650, 1615, 1400, $1266 \mathrm{~cm}^{-1}$; MS $\left(\mathrm{FAB}^{+}\right) \mathrm{m} / \mathrm{z}: 371[\mathrm{M}+$ $1]^{+}$. Anal. calcd for $\mathrm{C}_{21} \mathrm{H}_{22} \mathrm{O}_{6}: \mathrm{C} 68.10, \mathrm{H}$ 5.99; found C67.92, H 6.11.

\subsection{7-O-异戊烯基-3'-O-甲基香叶木素(11)的合成}

称取 $1(108 \mathrm{mg}, 0.34 \mathrm{mmol})$, 无水 $\mathrm{K}_{2} \mathrm{CO}_{3}(0.19 \mathrm{~g}$, $1.36 \mathrm{mmol}$ )加入到 $50 \mathrm{~mL}$ 单口瓶中, 再加入 $15 \mathrm{~mL}$ 无水 丙酮, $50{ }^{\circ} \mathrm{C}$ 下搅拌 $1 \mathrm{~h}$, 缓慢滴加异戊烯基溴 $(0.06 \mathrm{ml}$, $0.50 \mathrm{mmol}$ )与 $3 \mathrm{~mL}$ 丙酮的混合溶液, TLC 跟踪反应至原 料点消失, 约 $3 \mathrm{~h}$ 后停止反应, 冷却至室温, 过滤, 用少 量丙酮润洗，滤液旋干，得到粗产物，硅胶柱层析分离 [洗脱剂: $V$ (石油醚) $: V($ 乙酸乙酯 $)=4: 1$ ] , 得亮黄色粉 末状固体 $125 \mathrm{mg}$, 产率 $95.2 \%$. m.p. $114 \sim 116^{\circ} \mathrm{C} ;{ }^{1} \mathrm{H}$ NMR $\left(\mathrm{CDCl}_{3}, 400 \mathrm{MHz}\right) \delta: 12.78(\mathrm{~s}, 1 \mathrm{H}, 5-\mathrm{OH}), 7.52(\mathrm{dd}$, $\left.J=8.4,2.0 \mathrm{~Hz}, 1 \mathrm{H}, 6{ }^{\prime}-\mathrm{H}\right), 7.33$ (d, $\left.J=2.0 \mathrm{~Hz}, 1 \mathrm{H}, 2^{\prime}-\mathrm{H}\right)$, 6.97 (d, $\left.J=8.4 \mathrm{~Hz}, 1 \mathrm{H}, 5^{\prime}-\mathrm{H}\right), 6.57$ (s, 1H, 3-H), 6.49 (d, $J=2.0 \mathrm{~Hz}, 1 \mathrm{H}, 6-\mathrm{H}), 6.37$ (d, $J=2.0 \mathrm{~Hz}, 1 \mathrm{H}, 8-\mathrm{H}), 5.49$ (t, $J=6.4 \mathrm{~Hz}, 1 \mathrm{H}, 2 \mathrm{2}-\mathrm{H}), 4.58\left(\mathrm{~d}, J=7.2 \mathrm{~Hz}, 1 \mathrm{H}, 1 "-\mathrm{CH}_{2}\right)$, 3.98, 3.97 (each s, each 3H, 4'-- $\mathrm{OCH}_{3}, 3^{\prime}-\mathrm{OCH}_{3}$ ), 1.82, 1.77 (each s, each $3 \mathrm{H}, 4^{\prime \prime}-\mathrm{CH}_{3}, 5^{\prime \prime}-\mathrm{CH}_{3}$ ); $\mathrm{MS}\left(\mathrm{FAB}^{+}\right) \mathrm{m} / z: 383$ $[\mathrm{M}+1]^{+}$. Anal. calcd for $\mathrm{C}_{22} \mathrm{H}_{22} \mathrm{O}_{6}: \mathrm{C} 69.10, \mathrm{H} 5.80$; found C 69.26, H 5.72 .

\subsection{7-O-法呢烯基-3'-O-甲基香叶木素(12)的合成}

12 的合成方法同 11, 以 1 和法呢烯基溴为原料, 得 黄色固体, 产率 $88 \%$. m.p. $54 \sim 56{ }^{\circ} \mathrm{C} ;{ }^{1} \mathrm{H} \mathrm{NMR}\left(\mathrm{CDCl}_{3}\right.$, $400 \mathrm{MHz}$ ) $\delta: 12.75$ (s, 1H, 5-OH), 7.52 (dd, $J=8.4,2.0$ $\left.\mathrm{Hz}, 1 \mathrm{H}, 66^{\prime}-\mathrm{H}\right), 7.33$ (d, $\left.J=2.0 \mathrm{~Hz}, 1 \mathrm{H}, 2^{\prime}-\mathrm{H}\right), 6.98$ (d, $J=$ $\left.8.4 \mathrm{~Hz}, 1 \mathrm{H}, 5{ }^{\prime}-\mathrm{H}\right), 6.58$ (s, 1H, 3-H), 6.49 (d, $J=2.0 \mathrm{~Hz}$, $1 \mathrm{H}, 6-\mathrm{H}), 6.37$ (d, $J=2.0 \mathrm{~Hz}, 1 \mathrm{H}, 8-\mathrm{H}), 5.49$ (t, $J=5.6 \mathrm{~Hz}$, $1 \mathrm{H}, 2 "-\mathrm{H}), 5.06 \sim 5.12$ (m, 2H, 6",10"-H), 4.61 (d, $J=6.4$ $\mathrm{Hz}, 1 \mathrm{H}, 1$ "'- $\mathrm{CH}_{2}$ ), 3.98, 3.97 (each s, each $3 \mathrm{H}, 4$ '- $\mathrm{OCH}_{3}$, 3'- $\left.\mathrm{OCH}_{3}\right), 2.12 \sim 2.16\left(\mathrm{~m}, 4 \mathrm{H}, 4 ", 5 "-\mathrm{CH}_{2}\right), 1.97 \sim 2.06(\mathrm{~m}$, $\left.4 \mathrm{H}, 8 ", 9 "-\mathrm{CH}_{2}\right), 1.77$ (s, 3H, 15"-- $\left.\mathrm{CH}_{3}\right), 1.67$ (s, 3H, $14 "-\mathrm{CH}_{3}$ ), 1.61, 1.59 (each s, each 3H, 12",13"-- $\mathrm{CH}_{3}$ ); MS $\left(\mathrm{FAB}^{+}\right) \mathrm{m} / z: 519[\mathrm{M}+1]^{+}$. Anal. calcd for $\mathrm{C}_{32} \mathrm{H}_{38} \mathrm{O}_{6}: \mathrm{C}$ 74.11, H 7.39; found C 74.31, H 7.28.

\subsection{5 合成化合物的生物活性测试}

采用 MTT 法对合成的化合物 1 12 进行体外抗肿 瘤细胞 HL-60(白血病细胞)、SMMC-7721(肝癌细胞), 
SK-BR-3(乳腺癌细胞)、A-549(肺癌细胞) 和结肠癌细胞 (SW480)的活性篎选. 使用 MTT(噻唑蓝)蛋白染色法. 以顺铂(MW300)和紫杉醇作为阳性对照.

实验方法: (1)接种细胞: 用含 10\%胎牛血清的培养 液(DMEM 或者 RMPI1640)配成单个细胞悬液, 以每孔 5000 10000 个细胞接种到 96 孔板, 每孔体积 $100 \mu \mathrm{L}$, 贴壁细胞提前 $12 \mathrm{~h}$ 接种培养. (2)加入待测化合物溶液 (固定浓度 $40 \mu \mathrm{mol} \cdot \mathrm{L}^{-1}$ 初篮, 在该浓度对肿瘤细胞生长 抑制在 $50 \%$ 附近的化合物设 5 个浓度进入梯度复篮), 每 孔终体积 $200 \mu \mathrm{L}$, 每种处理均设 3 个复孔. (3)显色: 37 ${ }^{\circ} \mathrm{C}$ 培养 $48 \mathrm{~h}$ 后, 每孔加 MTT 溶液 $20 \mu \mathrm{L}$. 继续睬育 $4 \mathrm{~h}$, 终止培养, 吸弃孔内培养上清液, 每孔加 $200 \mu \mathrm{L}$ 的 SDS 溶液 $(10 \%)$, 过夜睬育(温度 $37{ }^{\circ} \mathrm{C}$ ), 使结晶物充分融解. (4)比色: 选择 $595 \mathrm{~nm}$ 波长, 酶联免疫检测仪 (Bio-Rad 680) 读取各孔光吸收值, 记录结果, 以浓度为横坐标, 细胞存活率为纵坐标绘制细胞生长曲线, 应用两点法 (Reed and Muench 法)计算化合物的 $\mathrm{IC}_{50}$ 值.

\section{2 结果与讨论}

以丰富易得的天然二氢黄酮类橙皮苷为原料, 经脱 氢反应得黄酮类地奥明. 黄酮结构中各个位置上的酚羟 基活性不同, 一般为 $\mathrm{C}^{7}-\mathrm{OH}>\mathrm{C}^{3}-\mathrm{OH}>\mathrm{C}^{5}-\mathrm{OH}$. 由于 4 位 羰基的影响, 5 位羟基的反应活性较低. 所以设计合成 路线时, 首先用苄基和甲基两种基团选择性的将 $3^{\prime}$ 位 $\mathrm{OH}$ 进行保护. $3^{\prime}$ 位- $\mathrm{OH}$ 经甲基化或芐基保护后的地奥明 极性较大, 分离较困难, 因此直接将得到的混合物进行 酸催化水解, 然后再分离提纯, 得到 3'- $O$-甲基香叶木素 (1)及 3'-O-芐基香叶木素(13).

地奥明苄基保护采用 $\mathrm{K}_{2} \mathrm{CO}_{3}$ 作为缩合剂, 在 $\mathrm{N}_{2}$ 气 氛下进行. 在实验过程中, 将不同温度及反应时间下得 到的实验结果进行了比较. 结果发现, 如果温度较低 $\left(50 \sim 60{ }^{\circ} \mathrm{C}\right)$, 则需要的反应时间较长, 温度过高 $(>110$ ${ }^{\circ} \mathrm{C}$ )或者反应时间过长均会造成副产物增加, 最终确定 反应温度为 $90 \sim 98{ }^{\circ} \mathrm{C}$, 反应时间 $3 \sim 4 \mathrm{~h}$. 芐基保护后, 未经分离提纯直接水解脱糖基, 转化率较高. 地奥明 $3^{\prime}$ 位 $\mathrm{OH}$ 的 $\mathrm{O}$-甲基化, 选用 $\left(\mathrm{CH}_{3}\right)_{2} \mathrm{SO}_{4}$ 作甲基化试剂. 实验 之初, 尝试用 DMF 作溶剂, 在弱碱 $\mathrm{K}_{2} \mathrm{CO}_{3}$ 存在的条件 下, $60 \sim 70{ }^{\circ} \mathrm{C}$ 反应, 反应生成了目标物, 但是由于地奥 明不会完全甲基化, 而 3'- $O$-甲基香叶木素和香叶木素 在硅胶柱层析分离时较困难. 因此, 尝试在 $\mathrm{NaOH}$ 溶液 体系中进行反应, $\mathrm{NaOH}$ 溶液可以溶解地奥明, 也可作 为碱, 脱去地奥明 $3^{\prime}$ 位酚羟基的氢. 反应温度控制在 60 70 ${ }^{\circ} \mathrm{C}$ 时, 反应副产物仍较多. 经过多次尝试, 最终 选用 $\left(\mathrm{CH}_{3}\right)_{2} \mathrm{SO}_{4}$ 作甲基化试剂, 在 3\% $\mathrm{NaOH}$ 溶液中, 控 制温度在 $10 \sim 25{ }^{\circ} \mathrm{C}$ 进行反应. 实验发现 $3^{\prime}-O$-甲基地奥
明的水溶性增强，而地奥明在酸性溶液中溶解度小. 利 用它们溶解度的差异, 反应结束后, 先将溶液调至酸性, 未反应的地奥明会析出，过滤除去地奥明，得到甲基化 的粗产物. 改进后的方法, 有利于水解后分离纯化得到 化合物 3'-O-甲基香叶木素.

在系列黄酮糖苷的合成中, 以适当保护的 $3^{\prime}-O$ - 芐 基香叶木素(13)以及 $3^{\prime}-O$-甲基香叶木素(1)作为黄酮苷 元与活化的糖基给体进行糖苷化反应. 糖基给体溴代乙 酰糖合成纯化后应充分干燥, 放入冰箱中低温避光保 存, 最好是制得后立即使用. 糖苗化反应采用的是相转 移催化法, 用四丁基溴化铵(TBAB)作相转移催化剂, 在 $0.25 \mathrm{~mol} / \mathrm{L} \mathrm{K}_{2} \mathrm{CO}_{3}$ 溶液及 $\mathrm{CHCl}_{3}$ 两相体系中反应, 澳 代乙酰糖不稳定, 在高温下容易分解, 反应过程中还容 易碳化, 因而反应时温度不能过高, 确定在 $45 \sim 50{ }^{\circ} \mathrm{C}$. 反应中用较弱的碱 $\mathrm{K}_{2} \mathrm{CO}_{3}$ 代替了强碱 $\mathrm{KOH}$ 或 $\mathrm{NaOH}$, 减少了溴代乙酰糖的水解. 相转移催化黄酮糖苷合成 法, 反应体系简单, 操作简便, 立体选择性好而且收率 也相对较高. 同时, 实验时也尝试了用无水丙酮及无水 DMF 的混合溶液作溶剂, 无水 $\mathrm{K}_{2} \mathrm{CO}_{3}$ 作缩合剂, TBAB 作相转移催化剂的合成方法, 但是与前一种方法相比副 产物相对较多, 产率也相对较低. 因此, 最终采用了在 $\mathrm{K}_{2} \mathrm{CO}_{3}$ 溶液及 $\mathrm{CHCl}_{3}$ 两相体系中进行糖苷化反应. 将合 成的黄酮乙酰基糖苷在甲醇钠/甲醇体系中脱掉乙酰基, 得到系列黄酮糖苷类, 3'- $O$-芐基香叶木素乙酰糖苷需要 先在 $\mathrm{Pd} / \mathrm{C}$ 的催化下氢解脱掉芐基, 再脱乙酰保护基, 最 后得到黄酮糖苷类 $\mathbf{2} \sim \mathbf{5}$.

利用氢核磁共振谱中糖的端基质子的偶合常数判 断苷键的构型是目前最常用的方法 ${ }^{[9,10]}$. 糖的六元环中, $\beta$ 型糖的 $\mathrm{H}^{1}, \mathrm{H}^{2}$ 都处于 $a$ 键, 它们之间的偶合常数 $J_{1,2}=7 \sim 10 \mathrm{~Hz}$, 而 $\alpha$ 型糖的 $\mathrm{H}^{1}, \mathrm{H}^{2}$ 分别处于 $e$ 键和 $a$ 键, 它们之间的偶合常数 $J_{1,2}=2 \sim 6 \mathrm{~Hz}$. 化合物 $\mathbf{2} \sim 5$ 端基质 子的化学位移分别为 $\delta 5.09,5.04,5.07,4.99$, 其偶合常 数 $J_{1,2}$ 依次为 $9.0,7.2,7.6,7.2 \mathrm{~Hz}$, 可以判断所有产物的 糖苷键均为 $\beta$ 构型.

由于香叶木素 $3^{\prime}$ 位羟基已经甲基化或苄基保护, 而 5 位羟基与 4 位羰基形成分子内氢键, 反应活性较低, 因此在用 3'- $O$-甲基香叶木素(1)和 3'- $O$-芐基香叶木素 (13)作底物, 进行异成烯基化和法呢烯基化时，反应选 择性的发生在 7 位. 反应采用了较简单的无水 $\mathrm{K}_{2} \mathrm{CO}_{3} /$ 无水丙酮体系，异戊烯基溴和法呢烯基澳的热稳定性 差, 对温度很敏感, 反应时先将底物在无水丙酮中溶解, $\mathrm{K}_{2} \mathrm{CO}_{3}$ 存在的条件下摚拌, 生成大量氧负离子后再缓慢 滴加异戊烯基澳或法呢烯基澳, 这样反应可在短时间内 以高收率完成. 黄酮苷元与异戊烯基澳或法呢烯基澳的 反应机理为亲核取代机理. 法呢烯基溴和异戊烯基溴均 
容易形成较稳定的碳正离子结构 $\left(\mathrm{C}^{+}\right.$和双键的 $p-\pi$ 共轭 效应), 与水接触也容易发生取代反应，因此在反应过 程中要避免反应体系中有水的存在, 反应所用试剂均需 经过无水处理.

在用 $\mathrm{Pd} / \mathrm{C}, \mathrm{H}_{2}$ 对 7-O-异戊烯基-3'-O-芐基香叶木素 进行脱茮基反应时, 希望得到的目标化合物是 7-O-异戊 烯基香叶木素, 但是反应结束后, 发现异戊烯基的双键 也被氢化变成单键, 得到化合物 7- $O$-异戊基香叶木素 (10).
将合成的化合物 1 12 用 MTT 法进行体外抗肿瘤 细胞生物活性笁选，结果见表 1. 半数抑制浓度 $\mathrm{IC}_{50}$ 值 表明，化合物 10 对白血病细胞(HL-60)、肝癌细胞 (SMMC-7721)、肺癌细胞(A-549)、乳腺癌细胞(MCF-7) 和结肠癌细胞(SW480)均具有明显的体外生长抑制活 性; 化合物 6 和 11 对肝癌细胞(SMMC-7721)、乳腺癌细 胞(MCF-7) 以及结肠癌细胞(SW480)表现出一定的抑制 活性.

表 1 化合物对不同肿瘤细胞株的半数抑制浓度 $\left[\mathrm{IC}_{50}\left(\mu \mathrm{mol} \cdot \mathrm{L}^{-1}\right)\right]$

Table 1 Half-inhibitory concentration $\left[\mathrm{IC}_{50}\left(\mu \mathrm{mol} \cdot \mathrm{L}^{-1}\right)\right]$ of different compounds to different tumor cell lines

\begin{tabular}{|c|c|c|c|c|c|}
\hline 化合物 & 白血病(HL-60) & 肝癌(SMMC-7721) & 肺癌(A-549) & 乳腺癌(MCF-7) & 结肠癌(SW480) \\
\hline 1 & $>40$ & $>40$ & $>40$ & $>40$ & $>40$ \\
\hline 2 & $>40$ & $>40$ & $>40$ & $>40$ & $>40$ \\
\hline 3 & $>40$ & $>40$ & $>40$ & $>40$ & $>40$ \\
\hline 4 & $>40$ & $>40$ & $>40$ & $>40$ & $>40$ \\
\hline 5 & $>40$ & $>40$ & $>40$ & $>40$ & $>40$ \\
\hline 6 & $>40$ & 22.05 & $>40$ & 21.23 & 20.44 \\
\hline 7 & $>40$ & $>40$ & $>40$ & $>40$ & $>40$ \\
\hline 8 & $>40$ & $>40$ & $>40$ & $>40$ & $>40$ \\
\hline 9 & $>40$ & $>40$ & $>40$ & $>40$ & $>40$ \\
\hline 10 & 16.24 & 5.80 & 10.35 & 4.70 & 8.55 \\
\hline 11 & $>40$ & 22.77 & $>40$ & 20.35 & 21.44 \\
\hline 12 & $>40$ & $>40$ & $>40$ & $>40$ & $>40$ \\
\hline 顺铂 & 2.51 & 14.99 & 13.61 & 18.65 & 18.85 \\
\hline 紫杉醇 & $<0.008$ & $<0.008$ & $<0.008$ & $<0.008$ & $<0.008$ \\
\hline
\end{tabular}

\section{References}

[1] Nakanishi, T.; Ogaki, J.; Inada, A.; Murata, H.; Nishi, M. J. Nat. Prod. 1985, 48, 491.

[2] Zhao, C.-C.; Shao, J.-H.; Cao, D.-D.; Zhang, Y.-W.; Li, X. China J. China Mater. Med. 2009, 34, 2761 (in Chinese). (赵春超，邵建华，曹丹丹，张玉伟，李铣，中国中药杂志，2009, 34, 2761.)

[3] Lin, L. Z.; Harnly, J. M. Food Chem. 2010, 120, 319.

[4] Desoky, S. K.; Hawas, U. W.; Sharaf, M. Chem. Nat. Compd. 2007, 43,542 .

[5] Epifano, F.; Curini, M.; Genovese, S.; Blaskovich, M.; Hamilton, A.; Sebti, S. M. Bioorg. Med. Chem. 2007, 17, 2639.

[6] Clark, M. K.; Scott, S. A.; Wojtkowiak, J.; Chirco, R.; Mathieu, P.;
Reiners, J. J.; Mattingly, R. R.; Borch, R. F.; Gibbs, R. A. J. Med. Chem. 2007, 50, 3274.

[7] Shan, Y.; Li, G.-Y.; Wang, Q.-A.; Li, Z.-H. Chin. J. Org. Chem. 2008, 28, 1024 (in Chinese).

(单阳, 李高阳, 汪秋安, 李忠海, 有机化学, 2008, 28, 1024.)

[8] Zhao, J.; Zhang, Z.-P.; Chen, H.-S.; Zhang, X.-Q.; Chen, X.-H. Acta Pharm. Sin. 1998, 33, 22 (in Chinese). (赵晶, 张致平, 陈鸿珊, 张兴权, 陈湘红, 药学学报, 1998, 33, 22.)

[9] Wang, Q.-A.; Wu, Z.; Liu, L.; Zou, L.-H.; Luo, M. Chin. J. Org. Chem. 2010, 30, 1682 (in Chinese)

(汪秋安, 吴峥, 刘莉, 邹亮华, 罗茗, 有机化学, 2010, 30, 1682.)

(Zhao, C.) 